This is the peer reviewed version of the following article: Riahinezhad, M., McManus, N., \& Penlidis, A. (n.d.). Shear Viscosity of Poly (Acrylamide/Acrylic Acid) Solutions. Macromolecular Symposia, 360(1), 179-184, which has been published in final form at https://doi.org/10.1002/masy.201500092 This article may be used for noncommercial purposes in accordance with Wiley Terms and Conditions for Self-Archiving.

\title{
Shear Viscosity of Poly (acrylamide/acrylic acid) Solutions
}

\author{
Marzieh Riahinezhad, Neil McManus, Alexander Penlidis*
}

Institute for Polymer Research (IPR), Department of Chemical Engineering, University of

Waterloo, Waterloo, ON N2L 3G1, Canada

E-mail: penlidis@uwaterloo.ca

\section{Summary}

Acrylamide (AAm)/Acrylic acid (AAc) copolymers are being used in a wide spectrum of applications, from which enhanced oil recovery (EOR) is the target application in this research study. The shear viscosity of the copolymer solution is extremely important in determining the performance properties of AAm/AAc copolymers. A series of copolymers with selected properties were prepared to study the effect of polymer concentration, copolymer composition, and salinity on the shear viscosity of the copolymer solutions. The results revealed the considerable effect of solution concentration and salinity on shear viscosity. Moreover, the behavior of the copolymer composition showed a maximum with respect to shear viscosity.

Keywords: acrylamide, acrylic acid, polyelectrolyte solution, shear viscosity, copolymerization 


\section{Introduction}

Copolymers of acrylamide (AAm) and acrylic acid (AAc) are imporatnt water soluble polymers used in numerous applications, such as drag reduction agents, paper and textile, biomedical, pharmaceutical, cosmetic products, flocculants for waste water treatment, and enhanced oil recovery $(\mathrm{EOR}) \cdot{ }^{[1-4]}$

Applications in EOR have received a lot of attention both scientifically and practically in recent years. In order to achieve the highest possible efficiency in EOR, it is critical to satisfy typical EOR requirements, such as high polymer solution viscosity, low polymer retention in the oil reservoir, and high mechanical, chemical and thermal stability. ${ }^{[5-8]}$ To satisfy these requirements, one has to modify copolymer properties, especially solution viscosity, and this is only possible through detailed understanding of the copolymerization kinetics (as copolymerization understanding will control copolymer chain microstructure).

Solution viscosity and behavior of AAm/AAc copolymers are extremely important in understanding and predicting the copolymer behavior in the specific application. For example, in applications such as flocculation, drag reduction or EOR, when the polymer is subjected to high shear stresses, degradation might happen and therefore having a detailed knowledge about the solution viscosity profile at different shear rates is necessary.

Shear viscosity of polyacrylamide and its degradation behavior have been studied by AbdelAlim et al. using a high shear Couette viscometer. ${ }^{[7]}$ They studied the shear degradation of polyacrylamide after exposing it to various shear stresses at different temperatures. Despite the fact that it was initially assumed that shear rate was the controlling factor, they concluded that shear stress and viscosity of the polymer were more important than shear rate. At constant shear rate, by decreasing the temperature and increasing the polymer solution concentration, both 
shear stress and polymer viscosity increase, which results in more degradation (and narrower molecular weight distributions).

Copolymer composition plays an important role in polymer solution behavior. Kulicke and Hörl observed a maximum in the solution viscosity profile of AAm and sodium acrylate (NaAc) copolymers with respect to copolymer composition. ${ }^{[9]}$ By increasing the AAm percentage in the copolymer from zero to $35 \%$, the solution viscosity went up. However, upon further increasing the AAm in the copolymer composition, the solution viscosity decreased. They also noticed a similar dependence on the AAm content for the radius of gyration of the copolymer. The authors explained this maximum based on the polyelectrolyte character of the copolymer (electrostatic effects) and also on non-ionic intermolecular hydrogen bonding. The electrostatic effect is the result of the AAm/AAc copolymer polyelectrolyte nature. AAc is a weak acid that tends to dissociate in water and therefore imparts negative charges on the copolymer chain. Because of these negative charges and the counter ions that surround the copolymer chain, there are electrostatic interactions in the aqueous solution which are responsible for the polyelectrolyte nature of the AAm/AAc copolymer.

The same maximum for AAm/AAc copolymer solutions was also reported by Myagchenkov et al. ${ }^{[10]}$ Based on their results of viscosity number of AAm and NaAc copolymers with respect to the degree of neutralization, near $70-80 \%$ degree of neutralization, there is a maximum for the viscosity number value because of the macroanion-counter ion interactions.

Besides shear viscosity, intrinsic viscosity also varies with copolymer composition. Candau et al. studied the intrinsic viscosity of $\mathrm{AAm} / \mathrm{NaAc}$ copolymer with respect to the $\mathrm{NaAc}$ content in the copolymer and observed a maximum in intrinsic viscosity of the solution around $40 \%$ (molar) of NaAc. ${ }^{[1]}$ They explained it based again on electrostatic interactions and hydrogen bonding within the copolymer chains. At NaAc contents below $40 \%$, because of cyclization between amide and carboxylate groups, chain stiffness and an increase in intrinsic viscosity happens. At NaAc content higher than $40 \%$, the probability of intramolecular hydrogen bonding 
decreases (due to high electrostatic repulsions between the chains), and subsequently the intrinsic viscosity decreases.

McCormick and Salazar reported a similar behavior for the copolymers of AAm and sodium 3acrylamido-3-methylbutanoate $(\mathrm{NaAMB}) .{ }^{[12]}$ They observed a maximum in the intrinsic viscosity of AAm/NaAMB copolymers with respect to copolymer composition. This behavior was explained based on counterion condensation and decrease in molecular weight when having more NaAMB in the copolymer. Moreover, the copolymer composition at which the intrinsic viscosity is at a maximum depends on hydrogen bonding (between the comonomer units of the copolymer backbone) that makes the copolymer chain stiff.

Solution viscosity of AAm/AAc copolymers with respect to polymer concentration has also been studied in the literature. ${ }^{[13-15]}$ Solution shear viscosity of hydrolyzed PAAm increases significantly with having more concentrated solutions at constant temperature. ${ }^{[13,16]}$

The other important factor that affects the rheology of AAm/AAc copolymer is solution salinity. ${ }^{[17]}$ Since this copolymer has a polyelectrolyte nature, any factor that influences the electrostatic interactions will change the solution and rheological properties as well. It has been observed that adding salts (such as sodium chloride) into a polyelectrolyte solution screens the negative charges on the copolymer chain and, as a result, significantly reduces the polymer solution viscosity. ${ }^{[13]}$ By increasing salt concentration from 0 to $1 \mathrm{wt} \%$, there is a noticeable decrease in the solution viscosity, especially at low shear rates. But after a critical salt concentration, the change in solution viscosity is negligible. This phenomenon is called "critical salinity" and occurs because of saturation in the screening effect of salt cations.

In our previous studies, copolymerization kinetics and microstructural properties of AAm/AAc copolymers have been systematically studied. ${ }^{[18-20]}$ Based on the established framework that we have developed between copolymerization kinetics and copolymer structure, we prepared a set of copolymers with various copolymer compositions in order to evaluate their shear viscosity 
at various conditions. This information on shear viscosity will be used to design tailor-made copolymers for EOR applications in immediate future steps.

\section{Experimental}

Monomers (AAm, electrophoresis grade, purity $\geq 99 \%$; AAc, purtiy 99\%), initiator $\left(4,4^{\prime}\right.$-azobis-(4-cyano valeric acid)), inhibitor (hydroquinone), and sodium hydroxide were purchased from Sigma-Aldrich, Oakville, Canada. Water was Millipore quality $\left(18 \mathrm{M} \Omega \mathrm{cm}^{-1}\right)$ and methanol was ACS grade from VWR, Mississauga, Canada. Sodium chloride for ionic strength adjustments was also ACS grade from Merck, Kirkland, Canada. Nitrogen gas from Praxair, Toronto, Canada (4.8 grade) was used for degassing of solutions.

Copolymers of AAm/AAc were prepared by free radical polymerization. The details of the polymerization procedure have been explained in our previous studies. ${ }^{[18-20]}$ Copolymerization conditions such as total monomer concentration and copolymerization $\mathrm{pH}$ were selected based on knowledge from our previous studies to make copolymers with specific properties. ${ }^{[20]}$ The ionic strength was kept constant during polymerization between all the runs by adding the required amount of sodium chloride salt. ${ }^{[19]}$ To give an example, the copolymerization conditions for one of the samples are as follows: initial mole fraction of AAm in the comonomer feed $\left(\mathrm{f}_{0 \mathrm{AAm}}\right)$ 0.75; $\mathrm{pH}$ level 5 ; total monomer concentration $1.5 \mathrm{~mol} \mathrm{~L}^{-1}$; corresponding cumulative copolymer composition (mole fraction) of AAm units in the copolymer chains (cum $\left.\mathrm{F}_{\mathrm{AAm}}\right)$ 0.77.

Precipitation in methanol and gravimetry were used to determine the masses of copolymer products and fractional monomer conversions. Elemental analysis (CHNS, Vario Micro Cube, Elementar) was used to determine copolymer composition. A stress-controlled cone and plate rheometer (AR2000, TA instruments) was used to measure the shear viscosity of samples at room temperature. Cone and plate (ETC steel) with a $40 \mathrm{~mm}$ diameter and $1^{\circ}$ angle were used for all shear viscosity tests. 
Copolymers were subsequently dissolved in high purity water or an aqueous solution of sodium nitrate, $\mathrm{NaNO}_{3}\left(0.2 \mathrm{~mol} \mathrm{~L}{ }^{-1}\right)$, and sodium phosphate, $\mathrm{NaH}_{2} \mathrm{PO}_{4} / \mathrm{Na}_{2} \mathrm{HPO}_{4}\left(0.01 \quad \mathrm{~mol} \mathrm{~L}^{-1}\right)$, with $\mathrm{pH}$ adjusted to 7 . This was done to evaluate the effect of salts on the shear viscosity of polymers as opposed to pure water. It is crucial to grind the polymer into a fine powder before dissolving it in water or brine and also add the powder gradually, since the viscosity of polymer solutions is very high (otherwise, homogeneous polymer solutions cannot be obtained). The polymer solution concentration was varied between 0.01 and $0.005 \mathrm{~g} \mathrm{~mL}^{-1}$ to check the effcet of polymer concentration on shear viscosity.

All shear viscosity measurements were replicated independently (two replicates) and average values are reported herein. For example, in Figure 1, the shear viscosity of AAm/AAC copolymer with cumulative copolymer coposition of AAm equal to 0.44 , cum FAAm $=0.44$, is shown for two independent replicates. As one can easily see, the shear viscosity profiles are very close and as a result the avearge of the two runs is reported further in the paper.

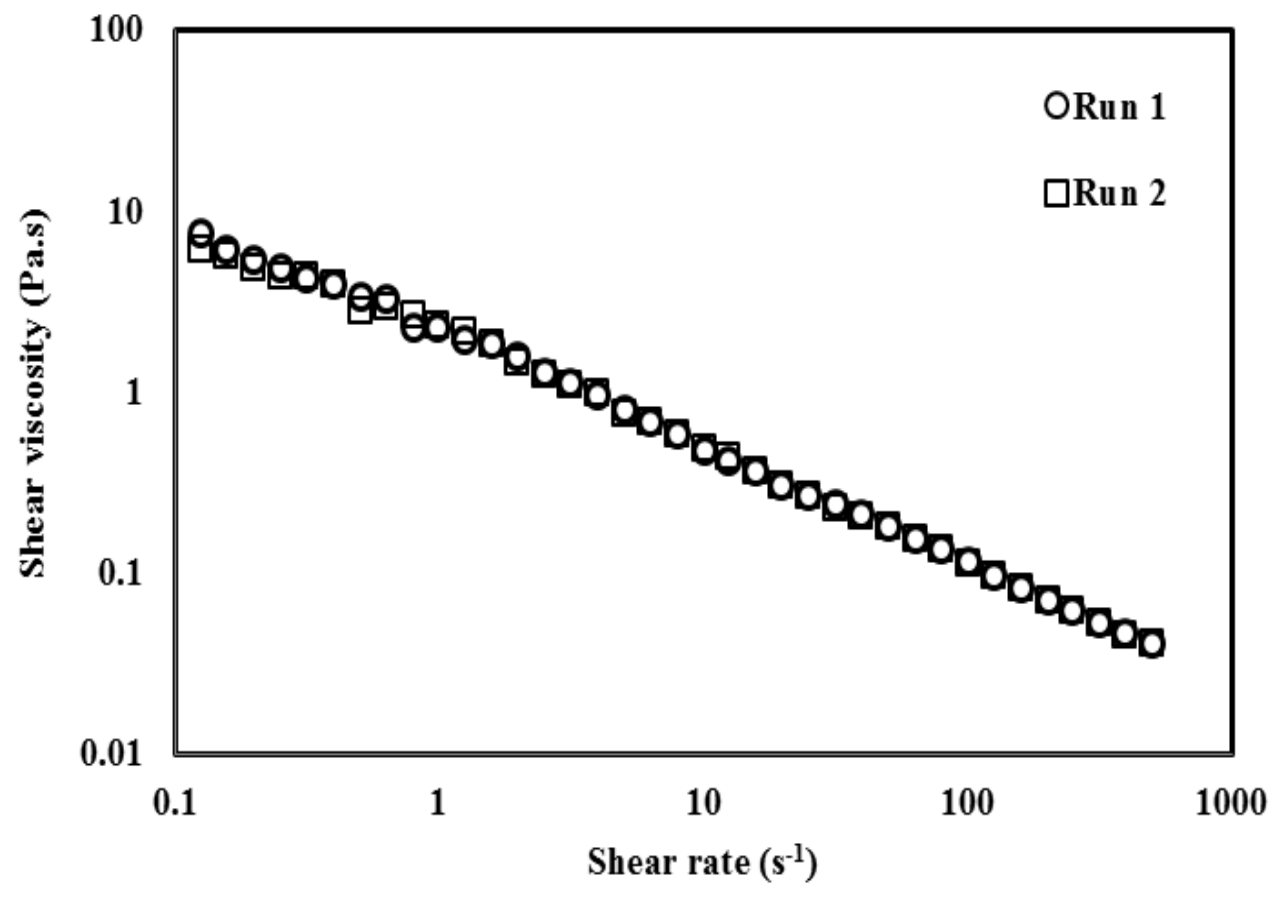


Figure 1. Shear viscosity of AAm/AAc copolymer aqueous solutions with cum $\mathrm{F}_{\mathrm{AAm}}=0.44$ and polymer solution concentration of $0.01 \mathrm{~g} \mathrm{~mL}^{-1}$, versus shear rate at $25^{\circ} \mathrm{C}$; two independent replicates.

\section{Results and Discussion}

The effect of polymer concentration on the shear thinning behavior of copolymer solutions was studied first. Figure 2 shows the shear viscosity of two AAm/AAc copolymer solutions of different polymer concentrations versus shear rate at $25^{\circ} \mathrm{C}$. Both polymer solutions exhibit shear thinning behavior, because of uncoiling and chain alignments with increasing shear rate, with an obvious increase of shear viscosity with higher copolymer concentration.

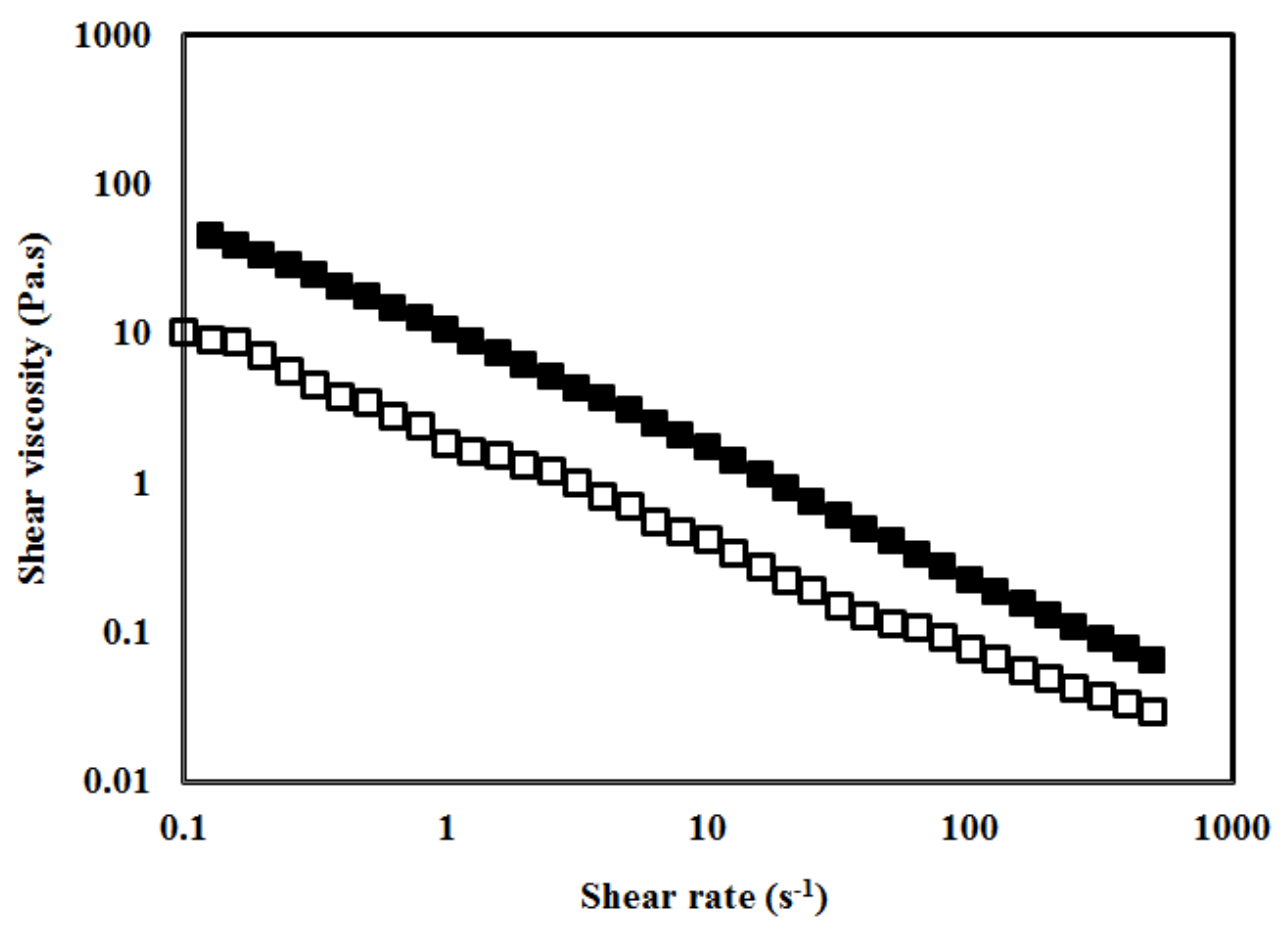


Figure 2. Shear viscosity of AAm/AAc copolymer aqueous solutions with cum $\mathrm{F}_{\mathrm{AAm}}=0.77$, versus shear rate, at two polymer solution concentrations: $0.01 \mathrm{~g} \mathrm{~mL}^{-1}$ (filled squares) and $0.005 \mathrm{~g} \mathrm{~mL}^{-1}$ (open squares) at $25^{\circ} \mathrm{C}$.

In order to check the effect of salinity on solution viscosity, two sets of polymer solutions were prepared in high purity water and buffer solution. The buffer solution was an aqueous solution of $\mathrm{NaNO}_{3}(0.2 \mathrm{~mol} \mathrm{~L}-1)$ and $\mathrm{NaH}_{2} \mathrm{PO}_{4} / \mathrm{Na}_{2} \mathrm{HPO}_{4}(0.01 \mathrm{~mol} \mathrm{~L}-1$ each $)$ with a total concentration of $0.22 \mathrm{M}$ at $\mathrm{pH}=7$. Representative results of shear viscosity versus shear rate are shown in Figure 3 to highlight the effect of the presence of salts in the solutions on shear viscosity. The polymer in buffer solution exhibited a noticeable reduction in shear viscosity compared to the polymer dissolved in pure water. In the presence of sodium nitrate and sodium phosphate salts, the negative charges on the copolymer chains and, consequently, the electrostatic repulsions within the polyelectrolyte solution are neutralized (collapse of the electrostatic fields surrounding the copolymer). Therefore, the polymer chain conformation changes from a stretched chain to a random coil structure. Hence, it can be concluded that by adding salts in the solution, the polymer coil dimensions and, subsequently, solution viscosity decrease. This is in agreement with what has been reported in the literature for this copolymer. ${ }^{[10,13]}$ 


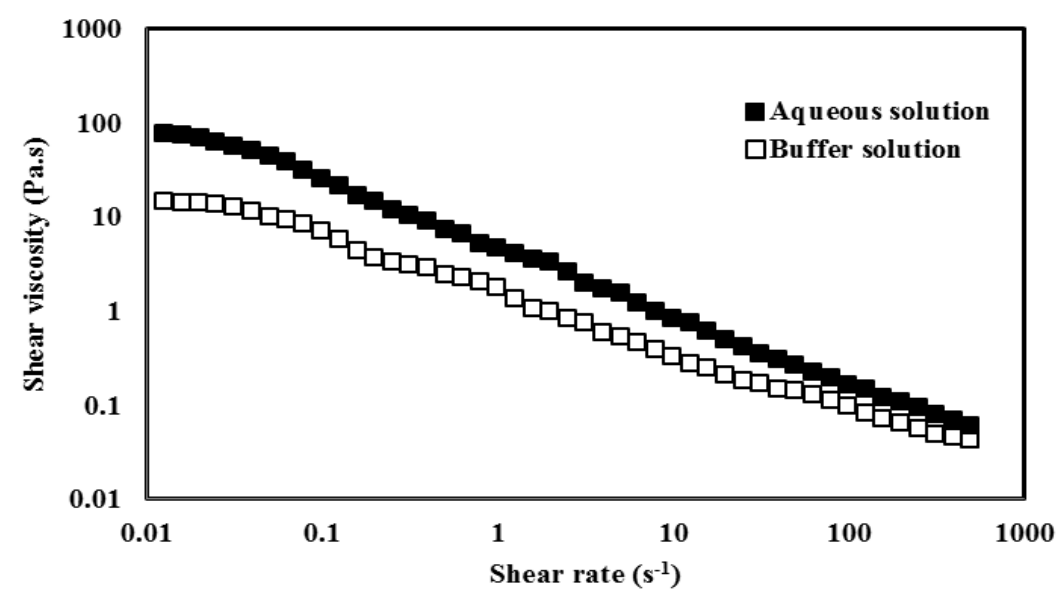

Figure 3. Shear viscosity of AAm/AAc copolymer solutions with cum $\mathrm{F}_{\mathrm{AAm}}=0.64$ and polymer solution concentration of $0.01 \mathrm{~g} \mathrm{~mL}^{-1}$; aqueous solution with high purity water (filled squares) and buffer solution with added salts (open squares) versus shear rate at $25^{\circ} \mathrm{C}$.

The other important factor that affects solution viscosity is copolymer composition. The shear thinning behavior of AAm/AAc copolymers with various copolymer compositions is shown in Figure 4. As can be seen from Figure 4, depending on the AAm fraction in the copolymer chains, different solution viscosity profiles arise.

At first glance, the shear viscosity profiles of Figure 4 look puzzling. As cum $\mathrm{F}_{\mathrm{AAm}}$ increases from 0.2 to 0.44 to 0.87 , shear viscosity values increase as well. But then an interesting reversal takes place at cum $\mathrm{F}_{\mathrm{AAm}}$ of 0.77 . Although the copolymer composition is lower than 0.87 , the shear viscosity is higher. The same observation holds for cum $F_{\text {AAm }}$ of 0.72 . Furthermore, the polymer with 0.96 AAm cumulative copolymer composition (mole fraction) exhibits viscosity values almost as low as the ones for cum $\mathrm{F}_{\mathrm{AAm}}=0.2$. 


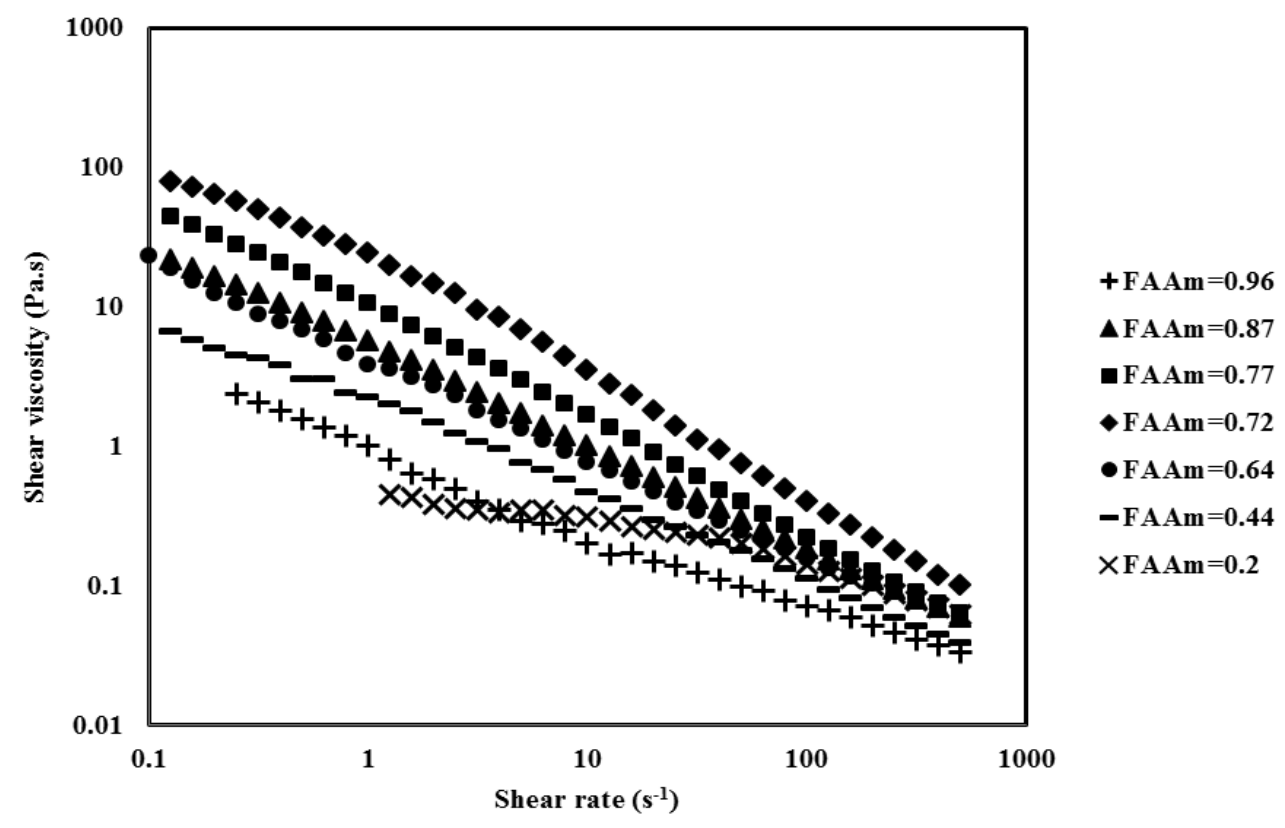

Figure 4. Shear viscosity of AAm/AAc copolymer aqueous solutions with polymer solution concentration $0.01 \mathrm{~g} \mathrm{~mL}^{-1}$ versus shear rate at $25^{\circ} \mathrm{C}$; effect of copolymer composition.

Since the target application of this copolymer is in EOR, the shear viscosity of these copolymers can more meaningfully be compared at a shear rate equal to $5\left(\mathrm{~s}^{-1}\right)$, which is a typical shear rate in oil fields. The results of this comparison are presented in Figure 5, and the behavior can explain the observations in Figure 4 discussed above. Based on Figure 5, it can be seen that the copolymer with AAm cumulative fraction of 0.72 exhibits the highest shear viscosity among the copolymers. Therefore, AAm/AAc copolymers with about 30\% AAc content provide the highest shear viscosity and are thus favorable in EOR because of their viscosity enhancement capability, which is one of the main reasons of polymer flooding. 


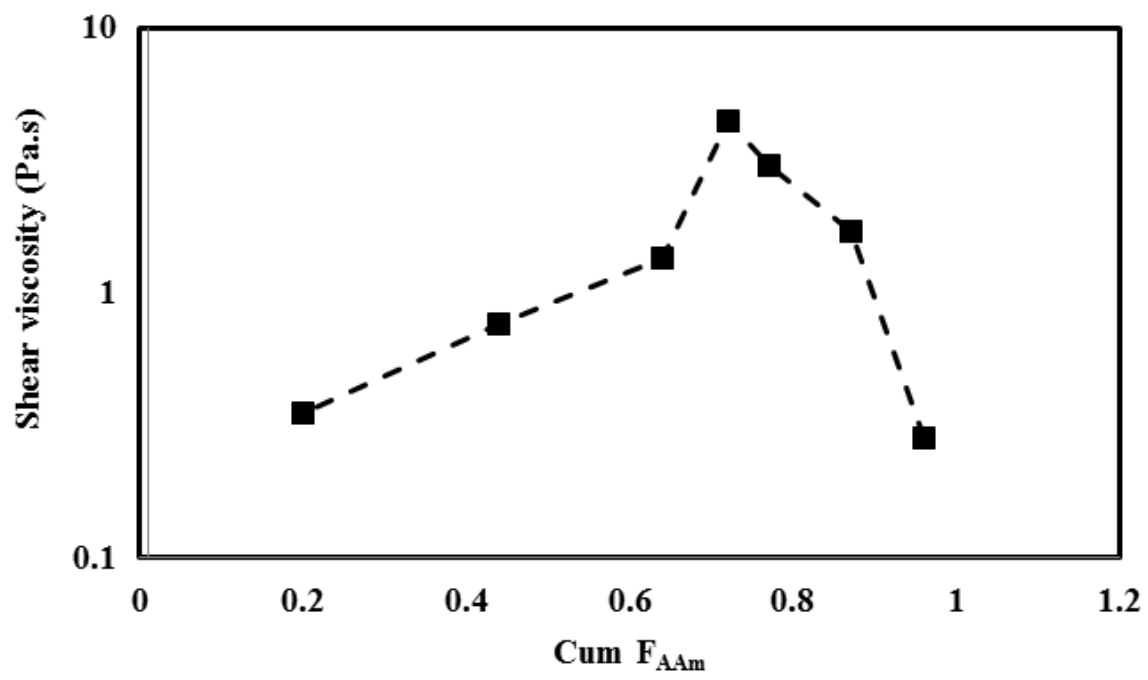

Figure 5. Shear viscosity of AAm/AAc copolymer aqueous solutions versus cumulative copolymer composition at fixed polymer solution concentration of $0.01 \mathrm{~g} \mathrm{~mL}^{-1}$ and $25^{\circ} \mathrm{C}$.

The copolymer composition changes the electrostatic environment of the copolymer, and as a result the copolymer chain size and solution properties will be different. By having more AAc (or less AAm) in the copolymer, more electrostatic interactions will be involved. These interactions extend the polymer chains and this increases the shear viscosity. In the presence of salts in the solution, these electrostatic repulsions are screened and therefore the polyelectrolyte nature does not play such a dominant role in the shear viscosity behavior of the solution. Instead, having more AAc enhances the chance of intramolecular hydrogen bonding and chain stiffness, and therefore increases shear viscosity of the polymer solution. On the other hand, having more AAm in the copolymer increases the copolymer molecular weight based on the results from our previous study. ${ }^{[20]}$ At typical copolymerization conditions, the peak average molecular weight of the copolymers can increase from 2.5 to 6.0 million, when the cumulative copolymer composition of AAm changes from 0.1 to 0.8 . These observations bring about a maximum effect of the copolymer composition on the shear thinning behavior of copolymer solutions. In other words, there is a specific copolymer composition at which the shear viscosity of the 
solution is maximum (see Figure 5), and the region of the maximum should be used in order to obtain the highest viscosity enhancement, as needed for EOR applications.

\section{Conclusions}

The shear viscosity of acrylamide (AAm)/acrylic acid (AAc) copolymers was studied. It was observed that having concentrated copolymer solutions enhanced the shear viscosity. In addition, shear viscosity decreased in the presence of salts in the polymer solution because of neutralizing the electrostatic repulsions between the chains. Moreover, the shear viscosity of AAm/AAc copolymer solutions showed a maximum around 30\% AAc (70\% AAm) in the copolymer. Hence, in order to achieve higher solution viscosity, which is required in EOR applications, one can make concentrated polymer solutions with copolymer composition around $30 \%$ AAc. In other words, by adjusting the copolymer composition and solution concentration, the shear viscosity of AAm/AAc copolymers can be maximized.

\section{Acknowledgments}

The authors acknowledge financial support from the Natural Sciences and Engineering Research Council (NSERC) of Canada, and the Canada Research Chair (CRC) program.

\section{References}

[1] C. A. Finch, Chemistry and Technology of Water-soluble Polymers, Royal Society of Chemistry (Great Britain); Plenum Press: 1983.

[2] J. M. Ebeling, K. L. Rishel, P. L. Sibrell, Aquacult. Eng. 2005, 33, 4, 235-249.

[3] V. A. Myagchenkov, V. F. Kurenkov, Polym. Plast. Technol. Eng. 1991, 30, 2, 109-135. 
[4] R. K. Will, J. Pearson, K. Yokose; U. Löchner; U. Fink, Synthetic Water-Soluble Polymers, 2011.

[5] J. M. Maerker, Soc. Petrol. Eng. 2012, 15, 4, 311-322.

[6] D. A. Z. Wever, F. Picchioni, A. A. Broekhuis, Prog. Polym. Sci. 2011, 36, 11, 1558-1628.

[7] A. H. Abdel-Alim, A. E. Hamielec, J. Appl. Polym. Sci. 1973, 17, 12, 3769-3778.

[8] A. Zaitoun, P. Makakou, N. Blin, R. S. Al-Maamari, A. A. Rashid Al-Hashmi, M. AbdelGoad, Soc. Petrol. Eng. 2012, 17, 2, 335-339.

[9] W. M. Kulicke, H. H. Hörl, Colloid Polym. Sci. 1985, 263, 7, 530-540.

[10] V. A. Myagchenkov, A. K. Vagapova, V. F. Kurenkov, M. A. Nagel, Polym. Sci. U. S. S. R. 1988, 30, 4, 759-764.

[11] F. Candau, Z. Zekhnini, F. Heatley, E. Franta, Colloid Polym. Sci. 1986, 264, 8, 676-682.

[12] C. L. McCormick, L. C. Salazar, J. Macromol. Sci., Part A 1992, 29, 3, 193-205.

[13] J. C. Jung, K. Zhang, B. H. Chon, H. J. Choi, J. Appl. Polym. Sci. 2013, 127, 6, 4833-4839.

[14] X. Y. Wu, D. Hunkeler, A. E. Hamielec, R. H. Pelton, D. R. Woods, J. Appl. Polym. Sci. 1991, 42, 7, 2081-2093.

[15] J. Klein, R. Heitzmann, Makromol. Chem. 1978, 179, 8, 1895-1904.

[16] M. Buchgraber, T. Clemens, L. M. Castanier, A. A. Kovscek, Soc. Petrol. Eng. Reservoir Eval. Eng. 2011, 14, 3.

[17] G. Muller, J. P. Laine, J. C. Fenyo, J. Polym. Sci. Polym. Chem. 1979, 17, 3, 659-672. 
[18] M. Riahinezhad, N. Kazemi, N. McManus, A. Penlidis, J. Polym. Sci. A Polym. Chem. 2013, 51, 22, 4819-4827.

[19] M. Riahinezhad, N. Kazemi, N. McManus, A. Penlidis, J. Appl. Polym. Sci. 2014, 131, 20.

[20] M. Riahinezhad, N. McManus, A. Penlidis, Macromol. React. Eng. 2015, 9 (2), 100-113. 